\title{
Improved extreme programming methodology with inbuilt security
}

\begin{abstract}
Security in e-commerce is becoming an important issue to deal with as several cases of attacks to users' data are increasing exponentially. Therefore, there is absolute need for proper checks and balances to mitigate the lapses using the appropriate development framework. This paper provides as a candidate, Improve Extreme Programming Methodology by tightening security controls across the development stages without necessarily extending the process.
\end{abstract}

Keyword: Development framework; E-commerce security; Extreme programming; Security; Web engineering 